\title{
Research on the Reconstruction Technology of the Computer Monitoring System of the Hydroelectric Power Plant in the Border Areas
}

\author{
Majia Luolun \\ Institute of Science and Engineering, Dehong Teachers'College, Mangshi, 678400, Yunnan, China \\ email: sunshine2325@163.com
}

Keywords: Hydropower Plant Planning, Computer Monitoring System, Transformation Technology

\begin{abstract}
The computer monitoring system of power generation equipment has very important economic and social benefits for stabilizing the production process of power plant, reasonable and economic production, improving labor productivity, improving production environment and reducing physical labor. Hydroelectric power station is a unit capacity of $35 \mathrm{~mW}$, with four large bulb tubular devices. The original monitoring system used the SAT 250 system of Austrian Ailin company. This paper discusses the current situation of the monitoring system of the complete power generation equipment, the development trend of computer technology and automatic control technology at home and abroad, and the operation management elements of the complete power generation equipment. Research on the conversion technology of computer monitoring system in power station. This paper discusses the necessity and possibility of system reconstruction based on the application of existing computer monitoring system in power station, and investigates the practical problems. Through the analysis and demonstration of various potential reorganization plans, it is finally determined to be practical and optimal. By enriching the existing equipment of the reconstruction plan, the reconstruction cost is saved as much as possible, the reconstruction cycle is shortened, and the economy is improved. At the end of the paper, the main technical problems of the conversion are discussed and summarized, and the conversion effect is analyzed. There are the same problems with the monitoring system of power stations, and there are many other power stations with bulb tubular units in China that need to be improved. The research results of this technology can also be used to reconstruct and build computer power monitoring system for these power stations. The transformation is more successful and takes fewer detours.
\end{abstract}

\section{Introduction}

The power station monitoring system was implemented in July 1999 and provided by the Austrian company Ailin. It uses a range of products produced by the Austrian company vatech Group [1]. The configuration software of the operator workstation is sat 250, the operating system is sun Solaris 2.5.1 x 86, and the LCU uses SK 1703 and am 1703 intelligent control units of SK 1703 series. Host computer system includes server and operator station, engineer station, remote roll gateway and printer. Each part of the system is connected by $10 \mathrm{~m}$ dual channel Ethernet[1]. The basic computer system consists of 10 local control units. Each local controller cooperates with four power generation equipment, switchyard, public equipment, communication controller and equipment level computer. The component is connected via token ring network, and the operator station accesses the token. In addition to the ring network, the communication controller communicates with the gateway machine of the host computer system through the serial communication port. The gateway machine then communicates with the central and remote controllers of the remote area.

\section{Research Status and Development Trend of Monitoring System Transformation}

With the rapid development of computer technology, network and automation technology, 
computer monitoring technology has been widely used in domestic and foreign hydraulic equipment. At present, the use of computer monitoring for automatic generation and optimal scheduling is an inevitable choice for the construction of hydropower stations. The automatic control technology and products of computer control are quite mature[2]. With the development of technology, reliability has been improved. The use of automation system not only reduces the number of electronic control cabinets, but also greatly improves the reliability of the system and simplifies the manual operation. At present, most of the computer monitoring systems of power generation equipment are designed according to the goal of "no staff on duty, staff of duty", in order to meet the needs of the development of power industry and improve the safe operation and automation level of hydraulic equipment. At the same time, in order to improve the basic management level of hydropower station. With the change of computer monitoring system in newwa hydropower station, new system structure, new control device, new software and other new technologies are constantly appearing[3]. At present, the computer monitoring technology of hydropower station mainly faces network, intelligence, humanization, software configuration and the control of who. Development direction.

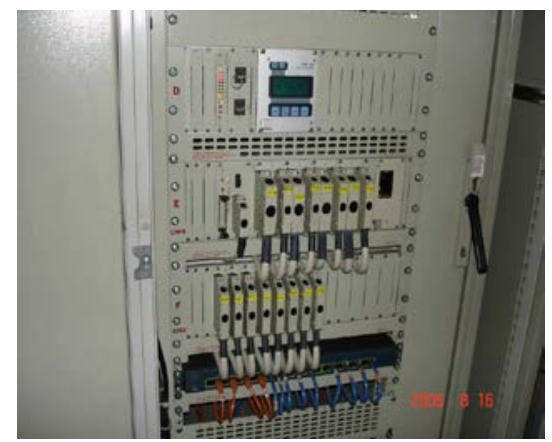

Figure 1 Cabinet diagram of computer monitoring system of Frontier Hydropower Station

\section{Computer Monitoring System for Power Plants Abroad}

ABB in Switzerland, Siemens in Germany, Ge in Canada and other foreign large-scale power plant computer monitoring system manufacturers are committed to the research, development and production of hydropower, thermal power, nuclear power computer monitoring system. Since the 1970s, many enterprises have adopted the latest computer system in the world, and applied it to the power station monitoring system, making its computer monitoring system always in the leading position in the world. The computer monitoring system produced by the former Austrian vatech company plays an important role in the world's hydropower and related industries[4]. There are also many application examples in tubular hydropower stations and substations in China.

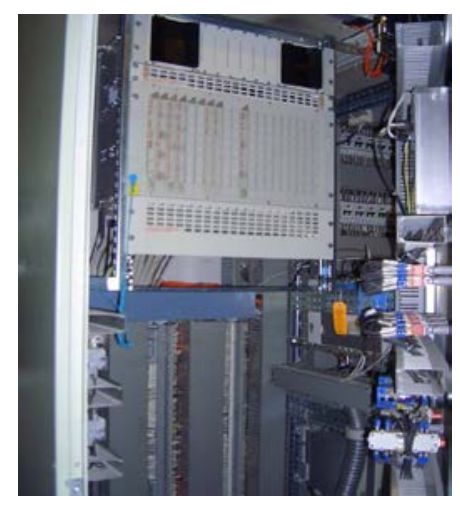

Figure 2 Site map of computer monitoring system of power station

\section{Computer Monitoring System of Domestic Power Plant}

Since the late 1960s, with the development and application of computer monitoring system in foreign power stations, China began to apply computer technology to the automation of power 
generation equipment. Especially after 1978, the application of computer technology and microprocessor single chip microcomputer has been deepened and promoted. China has not only introduced advanced foreign power station monitoring system, but also greatly improved the level of power station automation in China. Today's integrated automation of the independent automation of power stations and the overall computer monitoring of the centralized control assembly line has made great progress in China and the results of computer monitoring of thermal power, hydropower and nuclear power stations. The computer monitoring system of power station has reached the world level in hardware, software and function. Among them, Nai automation and the computer monitoring system produced by the Chinese Academy of water resources have always been the leaders in the domestic hydraulic automation industry. In June 2006, the MB 80 / MB 40 intelligent programmable controller produced by Nari passed various strict tests of electronic certification group company based in the United States[5]. All indicators of product test have passed CE certification and obtained EU market access certificate.

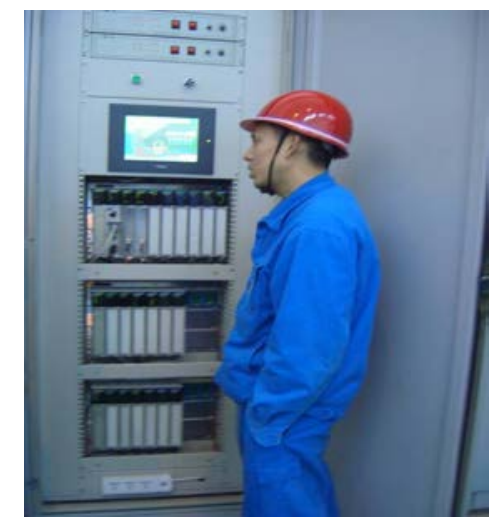

Figure 3 Site map of computer monitoring system of hydropower station

\section{Investigation on the Reconstruction of Monitoring System of Relevant Hydropower Stations in China}

In August 2006 and March 2007, a computer monitoring and reconstruction survey was carried out for wizhou hydropower station and Hunan power station near Sichuan Zipingpu, slongzhuuan, Xiaotiandu, Mintai and other hydropower stations. The respondents compared the software of network equipment conversion and computer monitoring system, as well as the hardware of SAT, Nari and zhushuike of three companies[6]. These are mainly for comprehensive understanding of the actual operation of each system of the power station, the possibility, method and effect of the conversion. It is of great reference significance and value to rebuild the computer monitoring system of Feilaixia Hydropower Station.

\subsection{Xiaolangdi Hydropower Station}

In 2005, the computer monitoring system produced by satellite company was partly reformed due to the problems of parts and components, and industrial Ethernet was used instead of snap ring network.

\subsection{Hunan Jinweizhou Hydropower Station}

In the first half of 2006, the modified sat computer monitoring system will replace the snap ring network with industrial Ethernet. The screen cabinet of its computer monitoring system is shown in Figure 1-2.

\subsection{Sichuan Huaneng Lengzhuguan Hydropower Station}

The site diagram of SAT computer monitoring system is shown. Before the transformation, the system is very similar to the situation of Feilaixia power station. The combination of PC workstation + UNIX + SAT250 is adopted for the upper computer, token ring network is adopted for the communication network, and sk1703 + am1703 is adopted for the lower computer[7]. In 
2005, the computer monitoring system of Lengzhuguan power station was reformed, the communication network was changed to optical fiber ring network, the sk1703 was changed to ak1703, the am1703 part was retained, and the equipment upgrade was realized.

\subsection{Xiaotiandu Power Station}

The scene of SAT computer monitoring system is shown. In addition, the lower computers are AK 1703 ACP and TM series products. The communication network adopts optical fiber network Ethernet, and two SCADA servers act as operator workstation / Engineer workstation.

\section{Transformation Principle}

The basic technical conditions of the computer monitoring system of the hydropower station, according to the operation and maintenance rules of the computer monitoring system of the hydropower station and the necessary conditions of the computer monitoring technology and testing technology of the rules of the modern hydropower station[8]. You can determine the principle of the transformation. The system is designed according to the principle of "no obligation employee", and the optimal configuration is designed according to the principle of safety, reliability, economy and practicability. In order to realize the functions of remote power supply, remote monitoring, remote control and remote coordination, communication is carried out between the power supply device and the power supply device. It is not only the high reliability local fault after the system conversion, but also the normal operation of the field equipment makes the various utilization performance indexes of MTBF, MTTR and other systems meet the basic technical requirements of the power saving of the computer monitoring system of the hydropower station. System configuration and equipment selection adapt to the rapid development of computers, and make the most of the advanced technology in the computer field. The system not only helps to expand functions and hardware, but also fully protects application resources and investment. This is a completely decentralized, completely open system. The design of distributed database and software module is suitable for the increase of function and scale. It can diagnose itself. Please ensure a smooth transition and seamless connection between the new computer monitoring system and the old one. The system meets the requirements of DL / T 5081-1997, DL/T 5065-1996 safety protection rules and regulations for computer monitoring system providing data network for power grid and power plant and relevant industrial technical standards. The system has high reliability, and local fault will not affect the normal operation of field equipment. The system's MTBF, MTTR, various indexes, "the basic technical conditions of the computer monitoring system of the hydropower station should be met."[9]. According to the actual needs of users, there are more specific performance indexes for special terms and conditions. In case of contradiction, two higher standards are the final conditions. The system has good real-time performance and strong interference and interference ability. , powerful function, convenient operation and simple maintenance. In addition, the performance of the transformed system is also very high. System composition and equipment selection, rapid computer development function, cutting-edge technology in the computer field, and the maximum use of the system are currently at a high level in the world. At present, the mainstream products are conservatively supporting the supply of spare parts for products. The rich and flexible background is to meet various conditions of customers. Easy to use editing and maintenance, software maintenance system, than simple. Moreover, this is the complexity of transformation, simplification, and shorter transformation period. Cost savings.

\section{Conclusion}

After the success of technology research, a change plan was made. The conversion completes the monitoring system in the shortest time. The conversion between the new system and the old system is smooth, the unit is closed in a short time, and the conversion process is greatly reduced[10]. The impact of operation ensures good economic benefits. Through the exchange of several hardware, the stability of the system device is ensured. Moreover, the operating life of the system has been 
greatly improved. After stopping, the hidden danger of the prepared parts of the system is excluded in the latest upper computer calculation, the problem of bad conservatism before application and the difficulty of data exchange of other systems such as management system.

\section{References}

[1] Didier Haguma, Robert Leconte, Stéphane Krau. Hydropower plant adaptation strategies for climate change impacts on hydrological regime. Canadian Journal of Civil Engineering, vol. 44, no. 11, 2017.

[2] F. Wang, Z. Qian, Z. Guo,. Pressure Oscillations Prediction of Axial Flow Pump with Adjustable Guide Vanes. Transactions of the Chinese Society for Agricultural Machinery, vol. 48, no. 3, pp. 119-123, 2017.

[3] Ablat X, Liu G, Liu Q, et al. Channel Wetlands Evolution Analysis From Liujiaxia to Togtoh County of Inner Mongolia in the Last Three Decades, 2017.

[4] Lindmark M, Olsson Spjut F. Perspectives on the Transformation of the Organic Energy System in 19th Century Sweden, 2017.

[5] Wei Ding, Betsy R. LaPlant, Timothy G. Call, et al. Pembrolizumab in patients with CLL and Richter transformation or with relapsed CLL, vol. 129, no. 26, pp. 3419-3427, 2017.

[6] Bourgin M, Beck B, Boehler M, et al. Evaluation of a full-scale wastewater treatment plant upgraded with ozonation and biological post-treatments: Abatement of micropollutants, formation of transformation products and oxidation by-products, 129, pp. 486, 2017.

[7] Ping Che, Ajith Anand, Emily Wu,. Developing a flexible, High-efficiency Agrobacterium mediated Sorghum Transformation System with Broad Application. Plant Biotechnology Journal, vol. 16, no. 7, 2018.

[8] Jing Xu, Fang Zhang, Chao Gao,. Transformation with Genes gag and gp120 from HIV-1 into Tomato and Regeneration of Transgenic Plant. Progress in Biotechnology, vol. 54, no. 3, pp. 37-40, 2017.

[9] Hau-Hsuan Hwang, Manda Yu, Erh-Min Lai. Agrobacterium -Mediated Plant Transformation: Biology and Applications. Arabidopsis Book, vol. 15, pp. e0186, 2017.

[10] Bowen Hu, Dawei Li, Xin Liu,. Engineering Non-transgenic Gynoecious Cucumber Using an Improved Transformation Protocol and Optimized CRISPR/Cas9 System, vol. 10, no. 12, pp. 1575, 2017. 